\title{
Personalidade de País e Auto Congruência: um estudo sobre o Brasil
}

\section{Country Personality and Self Congruence: a study on Brazil}

Personalidad de País y Auto Congruencia: un estudio sobre Brasil

\author{
Roger Barbara Silva ${ }^{1}$ \\ Janaina Moura Engracia Giraldi ${ }^{2}$
}

\section{Resumo}

O propósito deste estudo foi investigar a personalidade do Brasil enquanto destino turístico e avaliar as relações entre essa variável com a auto congruênciaque um grupo de estrangeiros tem com o país. Foi usada uma pesquisa descritiva quantitativa, por meio de um levantamento com 95 respondentes. As técnicas de análise empregadas foram a análise fatorial exploratória e a comparação de médias. Os resultados obtidosmostraram que, para os respondentes, as características da personalidade do Brasil podem ser agrupadas em cinco dimensões: Vibração, Competência, Contemporâneo, Sofisticação e Sensual. Esta última dimensão constitui em uma das principais contribuições deste artigo. Outra importante contribuição deste estudo para a literatura apresentou-se em relação à auto congruência e às dimensões da personalidade. Nessa relação, a intenção era analisar se, de acordo com nível de auto congruência (alta ou baixa) por parte dos respondentes, haveria diferença na pontuação das dimensões de personalidade do Brasil. O estudo concluiu que o nível de auto congruênciainfluência nos escores atribuídos nas dimensões da personalidade do país, entretanto não muda a ordenação das dimensões.

Palavras-Chave: Personalidade do destino turístico; Auto congruência; Imagem de destino; Brasil.

\begin{abstract}
The purpose of this study was to investigate the personality of Brazil as a tourism destination and to evaluate the relationship between this variable and the self congruity a group of foreigners has toward the country. A quantitative descriptive research was used, through a survey of 95 respondents. The analytical techniques employed were exploratory factor analysis and the comparison of means. The results obtained indicated that, to the respondents, the personality characteristics of Brazil can be grouped according to five dimensions: Vibration, Competence, Contemporary, Sophistication and Sexy. The latter dimension is one of the main contributions of this research. Another important contribution of this study to the literature is in regard the toself congruity and to the dimensions of personality. In this analysis, the intention

\footnotetext{
${ }^{1}$ Graduando em Administração pela Faculdade de Economia, Administração e Contabilidade da Universidade de São Paulo (FEA-USP), Ribeirão Preto, São Paulo. Brasil. E-mail: rogerbarbarasilva@ outlook.com

${ }^{2}$ Doutora em Administração pela Universidade de São Paulo. Professora Associada de Administração e Marketing da Faculdade de Economia, Administração e Contabilidade da Universidade de São Paulo, Ribeirão Preto, São Paulo. Brasil. E-mail: jgiraldi@usp.br
} 
was to verify whether, according to level of self congruity of the respondents (high or low), there would be differences in the scores of Brazil personality dimensions. The study concluded that the level of self congruity does influence on the scores assigned to the country's personality dimensions, however it does not change the dimensions ranking.

Keywords: Tourism destination's personality; Self congruity; Destination image; Brazil.

\section{Resumen}

El propósito de este estudio fue investigar la personalidad de Brasil como destino turístico y evaluar la relación entre esta variable con la auto congruencia que tiene un grupo extranjero con el país. Se utilizó un estudio descriptivo cuantitativo, a través de una encuesta de 95 encuestados. Las técnicas de análisis utilizadas fueron el análisis factorial exploratorio y la comparación de los promedios. Los resultados mostraron que para los encuestados, las características de personalidad del Brasil se pueden agrupar en cinco dimensiones: Vibración, Competencia, Contemporáneo, Sofisticación y Sensual. La última dimensión es una de las principales aportaciones de este artículo. Otra contribución importante de este estudio para la literatura es en relación con el auto congruencia y las dimensiones de la personalidad. En este sentido, la intención era examinar si, de acuerdo con el nivel de auto congruencia (alta o baja) de los encuestados, habría diferencia en las puntuaciones de las dimensiones de la personalidad de Brasil. El estudio encontró que el nivel de auto congruencia influye en las puntuaciones atribuidas en dimensiones de la personalidad del país, pero no cambia el orden de las dimensiones.

Palabras clave: Personalidad del destino turístico; Auto congruencia; Imagen de destino; Brasil.

\section{Introdução}

A imagem de um país é um importantefator que pode elevar o número de turistas no país. Segundo Kotler et al. (1993) a imagem de um país é definida como a soma de crenças e impressões das pessoas em referência ao país. O desenvolvimento de uma imagem confiável é fundamental para que os destinos turísticos desse país, bem como seus produtos e serviços se consolidem no mercado internacional e entrem na lista de opções a serem consideradas pelos turistas (PÉREZNEBRA; ROSA, 2008). A imagem é considerada um componente importante na escolha de um destino turístico (REZENDE-PARKER; MORRISON; ISMAIL, 2003; MARIUTTI; GIRALDI; CRESCITELLI, 2013). As crenças sobre um país e suas pessoas, ou seja, sua imagem, possuem uma influência direta nas crenças e avaliações de um destino (NADEAU et. al, 2008), isto é, a imagem de um país afeta diretamente a imagem do destino turístico.

No entanto, muitos destinos turísticos ainda prometem atributos similares uns aos outros, como cenários belos, praias exuberantes, oceanos lindos, ou lugares amigáveis como vantagens 
(ENKINCI et al., 2007, MURPHY; BENCKENDORFF; MOSCARDO, 2007). Contudo, esses atributos não configuraram em grande valor, visto que os mesmos não são grandes influenciadores na hora de o turista tomar a decisão de visitar aquele lugar, atribuindo apenas características naturais e básicas na imagem do destino.

Uma alternativa para auxiliar no desenvolvimento de estratégias de posicionamento eficazes, de modo a aumentar as vantagens competitivas de um destino, é identificar as dimensões de sua personalidade, que está relacionada ao fato de o destino possuir uma série de características humanas e essas associações podem afetar as intenções dos compradores/turistas em relação a um país específico (ROJÁS-MENDEZ, MURPHY; PAPADOPOULOS, 2013).

Ekinci e Hosany (2006) definem a personalidade do destino como um conjunto de traços de personalidade associados com esse destino. Esse conceito possui grande importância para este artigo, uma vez que pode auxiliar um destino a ser relevante para o turista e para que este se sinta atraído por ele. Ekinci e Hosany (2006) sugerem que a personalidade do destino pode ser usada como uma variável importante para a escolha do turista quanto ao lugar preferido.Neste aspecto, o uso de traços humanos para qualificar as decisões e ações dos países encoraja a personificação desses destinos (D'ASTOUS; BOUJBEL, 2007). Os traços de personalidade fornecem ricos significados, que permitem prever diferentes tipos de ações (D'ASTOUS; BOUJBEL, 2007), inclusive de compra e viagens.Dentro dessa ótica, a tentativa de associar o país com as características da personalidade humana pode ajudar os gestores a focar o seu público-alvo ou até mesmo mudar a imagem do país para que o seu público também se altere. O Brasil claramente tem um grande potencial no que se refere ao turismo, no entanto, este potencial não é totalmente atingido. Os meios de atração da nação brasileira ainda estão muito focados em futebol, carnaval, belezas naturais e alegria do povo brasileiro, que são importantes para o turismo, mas não bastam para que o potencial turístico seja atingido. Para melhorar essa imagem, é preciso compreender como o turista vê o Brasil.

Outro termo relevante para este artigo é o auto conceito, que tem avançado como uma construção útil para o entendimento e explicação do comportamento de escolha do consumidor. Tem-se sugerido que os consumidores escolhem seus produtos ou marcas que se assemelham ao consumidor ou mesmo ao modo como ele gostaria de parecer (LANDON, 1974; MALHOTRA, 1988; SIRGY, 1982). A auto congruência pode ser considerada uma extensão natural de auto 
conceito, tendo como hipótese básica o fato de um consumidor tender a selecionar produtos ou marcas que correspondem ao seu próprio auto conceito (USAKLI; BALOGLU, 2011).

Assim, o problema de pesquisa proposto neste artigo é: "Se o Brasil fosse uma pessoa, quais seriam seus elementos de personalidade"? O objetivo é investigar a personalidade do Brasil enquanto destino turístico e examinar a relação entre essa variável e a auto congruência do respondente. Para tanto, foi empregada uma pesquisa descritiva quantitativa junto a 95 respondentes estrangeiros, usando-se as técnicas estatísticas de análise fatorial exploratória e comparação de médias.

O conhecimento sobre as características mais relacionadas à personalidade de um país, principalmente, relacionadas ao Brasil, auxiliará as autoridades ligadas ao setor de turismo a reforçar a características salientadas, ou desistir daquelas não tão bem sucedidas, e criar as que foram destacas pelos turistas. Dessa forma, esta pesquisa pode se apresentar como uma ferramenta relevante para se criar valor ao turista que vem ao país, aumentar a retenção dos que já estão e atrair os que não conhecem e principalmente orientar os órgãos turísticos brasileiros a posicionar melhor suas estratégias.

\section{Imagem e Personalidade do Destino}

Embora o desenvolvimento das áreas de pesquisa sobre imagem de destino e imagem de país tenha ocorrido separadamente, ambas investigam avaliações complexas relacionadas a lugares e como esses afetam decisões (NADEAU et al, 2008). Segundo Beerli e Martín (2004), imagem de destino é o conceito formado pela interpretação emocional de diferentes aspectos turísticos. As pessoas preferem viajar para determinados lugares devido às boas imagens que as mesmas possuem daquele destino, por isso, é de suma importância saber administrar a percepção dos turistas em relação ao país (ASTOUS; BOUJBEL, 2007).

Dentro do aspecto de imagem, outro importante componente é o elemento afetivo. De acordo Baloglu e McCleary (1999), o principal fator que indica uma extensa disparidade na visão global de quatro destinos turísticos constitui-se no elemento afetivo, ao invés de outros, como a imagem cognitiva. Esta, por sua vez, de acordo com Lee e Xie (2011) e Prayag (2009), captura crenças e conhecimento sobre atributos físicos do destino. Por outro lado, de acordo com Baloglu e McCleary (1999) e Russel e Pratt (1980), a imagem afetiva de um destino turístico 
pode ser utilizada para explicar as intenções de comportamento em relação a certo lugar. Ekinci e Hosany (2006) realizaram um trabalho relacionando a imagem afetiva com a personalidade do destino, considerando este último um ótimo indicador de intenção para a recomendação de um destino para outros.

De acordo com Astous e Boujbel (2007), existem principalmente duas razões para demonstrar a importância dos estudos sobre personalidade de destinos. A primeira é que a apresentação dos países é feita frequentemente com traços humanos. Por exemplo, nos editoriais de jornais e revistas que citam esses países como agressivos, moralmente decadentes ou racistas. Em segundo Lugar, características humanas fornecem ricos significados e esses significados por sua vez permitem atribuir diferentes tipos de ações consistentes.

O temapersonalidade de destino começou a ser abordado com mais profundidade após os estudos de personalidade das marcas feitos por Aaker (1997), que buscou relacionar as marcas a características humanas, visto que os consumidores veem frequentemente as marcas com traços humanos. O autor conseguiu chegar a cinco principais traços de personalidade (sinceridade, excitação, competência, sofisticação e robustez). O trabalho de Aaker (1997), no entanto, segundo Ekinci e Hosany (2006), apresenta algumas lacunas quando relacionado ao setor de destinos turísticos, sendo mais voltado para a categoria de produtos. A personalidade do destino pode ser definida como um conjunto de traços de personalidade associados com o destino (EKINCI; HOSANY, 2006).

As dimensões de personalidade extraídas no estudo de Usakli e Baloglu (2011) vêem contribuir com a conclusão estabelecida por Hosany, Ekinci eUysal(2006), que sugere que alguns itens do estudo de personalidade da marca de Aaker (1997) são redundantes e não são totalmente coerentes para destinos turísticos. De forma similar ao estudo proposto por Aaker (1997), cinco dimensões depersonalidade emergiram em relação à cidade de Las Vegas. Em suas conclusões, Ekinci e Hosany (2006) estabeleceram que os destinostêm personalidades que refletem características comosinceridade, excitação e convivência. Dentre os traços de personalidade obtidos, quatro foram coerentes e convergiram para o quadro de personalidade da marca proposto por Aaker (1997). Isso indica que os resultados encontrados por Usakli e Baloglu (2011) podem ser aplicados ao setor de turismo, mas com suas devidas proporções, pois conforme observado o quinto fator (vibrante), está especificamente ligado com Las Vegas, explicando grande parte da variância do estudo. 
Na mesma direção, Astous e Boujbel (2007) propuseram uma escala para a personalidade do destino que incluía seis dimensões: agradabilidade, maldade, esnobismo, assiduidade, conformidade e moderação. Mais recentemente, Kim e Letho (2013) usaram uma "mistura" de métodos para explorar os traços de personalidade da Coreia do Sul, com foco nas diferenças entre a imagem do destino que o turista possui antes de visita-lo e a personalidade percebida. Os autores basearam-se na escala BPS (Brand Personality Scale) usada por Aaker (1997), que atribui cinco dimensões da personalidade a marca (sinceridade, excitação, competência, sofisticação e robustez). Entretanto, o estudo feito evidenciou duas novas dimensões da personalidade, relacionadasà Coreia do Sul: Única e Orientação Familiar.

Para embasar a discussão da personalidade do destino, alguns resultados serão pontuados acerca de casos que levam em consideração tal conceito. O primeiro resultado apresentado é o de Las Vegas, em que o questionário foi desenvolvido em parceria com administradores dos centros de convenções de Las Vegas e autoridades turísticas, e aplicado em turistas do referido destino. Com objetivo de encontrar traços de personalidade convergentes com a cidade, foram desenvolvidos dois estágios. O primeiro teve livre elucidação, ou seja, permitia identificar traços únicos da personalidade da cidade e, em segundo lugar, para validar a escala BPS foram utilizados 42 traços de personalidade.

No primeiro estágio, em que os respondentes tinham livre escolha para citar os traços de personalidade que lhes vinham à cabeça, esses foram solicitados a pensar se Las Vegas fosse uma pessoa, qual tipo de pessoa seria? A amostra foi feita com 58 pessoas. As respostas mais comuns foram: excitante $(15,54 \%)$, sexy $(14,50 \%)$, enérgica $(10,36 \%)$, vibrante $(9,32 \%)$, independente $(9,32 \%)$, única $(8,29 \%)$, viva $(8,29 \%)$, vistosa $(8,29 \%)$ e desobediente $(8,29 \%)$.

No segundo estágio, realizou-se uma análise de conteúdo, como forma de validar o BPS. Foram dispostos 42 traços de personalidade, em que os respondentes, foram avaliando e identificando esses traços de personalidade com Las Vegas. A escala usada indicava que a frase (1) não descreve o destino e (5) extremamente descritivo do destino. Dessa forma, chegou-se a um conjunto de 23 itens, que foram realocados em cinco dimensões: Sinceridade (original, amigável e alegre), Contemporâneo (legal, jovem, imaginativa, única, independente, contemporânea, acima da média, espirituosa e ousada), Competência (inteligente, sucesso, líder e confidente), Sofisticação (alta classe, glamorosa, boa aparência, charmosa e feminina) e Vibrante (enérgica, viva, excitante, sexy, vibrante e ousado). 
Os resultados mostrados acima indicam que os entrevistados foram levados e instigados a pensar na personalidade do destino e relacionar a mesma com características humanas, e até mesmo relacionar a cidade de Las Vegas com a sua própria personalidade, configurando uma tentativa de personificação e de alinhamento da auto congruência entre a cidade e o turista. Outra observação é a aproximação desses resultados com as dimensões feitas por Aaker (1997): sinceridade, excitação, competência, sofisticação e robustez.

Tendo como base os resultados de Usakli e Baloglu (2011), verifica-se que a personalidade do destino tem um impacto positivo na intenção de retorno e na recomendação do lugar turístico. Tais conclusões entram em convergência com pesquisas anteriores (EKINCI; HOSANY, 2006; EKINCI et al, 2007). Por conseguinte, outro resultado de grande relevância é em relação à teoria da auto congruência, que se conjuntura por apresentar a relação da personalidade do lugar e a do turista, demostrando que a auto congruência tem um impacto positivo no comportamento dos turistas, fazendo com que o mesmo tenha uma atitude favorável em relação a determinado destino turístico, resultando na intenção de voltar e no "boca a boca". Portanto, o estudo evidenciou o papel de mediador parcial que a auto congruência possui em relação à personalidade do destino e intenções comportamentais. Em outras palavras, a personalidade do destino tem um efeito indireto positivo na intenção de retorno e na recomendação, através da auto congruência.

No entanto, Papadimitriou, Apostolopoulou e Kaplanidou (2013) dissertam que ainda parece incerto como a personalidade do destino contribui para a formação da imagem global do destino e como influencia os resultados que são mensuráveis, como intenções de retorno ao destino e ou a vontade de citar o lugar de forma positiva. Alinhados com este pensamento, Lee e Suh(2011),Sahin e Baloglu(2011) e Usakli e Baloglu(2011) atribuem esta incerteza na escassez de investigações focadas no turismo urbano com o objetivo de examinar a personalidade das cidades destinos.Tais colocações se tornam de extrema importância para a presente pesquisa, pois espera-se conseguir analisara personalidade de um destino específico (Brasil), verificando quais dimensões da personalidade emergem. Destaca-se que a literatura também indica que há ainda falta de um conjunto confiável e válido para medição das dimensões da personalidade de país (ROJAS-MÉNDEZ; MURPHY; PAPADOPOULOS, 2013), sendo esta uma contribuição adicional desta pesquisa.

A personalidade do destino contribui de certa forma para a visualização de características humanas associadas ao país. A partir dessa atribuição, é possível associar essas personalidades 
a determinados indivíduos. No entanto, para que isso possa ocorrer, cada pessoa deve conhecer suas próprias características de personalidade, para futuramente observar ou desejar uma possível convergência com a personalidade de um destino turístico.A teoria de auto congruência fornece subsídios para que tal alinhamento seja feito.

\section{Auto Congruência}

Há uma estrita relação da personalidade com a teoria da auto imagem, em que pessoas preferem objetos que são congruentes com a sua personalidade (GRAEFF, 1996, ONKVISIT; SHAW, 1987). Consequentemente, atribuindo essa teoria ao turismo, pode-se inferir que as pessoas tendem a escolher os lugares que visitarão que mais se aproximam da sua personalidade.

De acordo com Usakli e Baloglu (2011) a hipótese básica na teoria da auto congruência é de que os consumidores tendem a selecionar produtos ou marcas que correspondem à sua própria personalidade. Por sua vez, Sirgyet al. (1997) dizem que o grau de consistência entre o auto conceito do consumidor e de determinada marca é referenciado como auto congruência. Sirgyet al. (1991) dissertam que a teoria da auto congruência propõe que o comportamento do consumidor é determinado, em partes, por um jogo cognitivo entre valor, expresso em atributos de um produto ou marca e auto conceito. Em referência a este jogo cognitivo, a teoria da auto congruência postula que a elevada preferência pela marca é reforçada pelas características simbólicas que se aproximam da auto percepção do indivíduo (SWANN; STEIN-SEROUSSI; GIESLER, 1992).

Epstein (1992) explica a necessidade de auto-congruência com um motivo principal "mantendo a coerência de um sistema conceitual pessoal" (p.35), ou seja, uma vez que este conjunto de crenças é estabelecido, indivíduos se esforçam para mantê-lo, por vezes, em face da angústia ou da falta de esforço externos. Para que esses conjuntos de crenças sejam estabelecidos, os indivíduos não só irão alterar o seu comportamento e interpretação de eventos para confirmar o auto conceito, mas vão incentivar o comportamento em outras pessoas, reforçando suas crenças em cima delas. Com isso, os consumidores preservam seu auto conceito, comprando produtos que os representam, ou indo a lugares turísticos que contribuam da mesma forma.

O estudo de Chon (1992), exemplifica de maneira prática a aplicação da auto congruência na indústria do turismo. Seu estudo foi realizado após o retorno da viagem com 225 indivíduos que haviam visitado Norfolk, Virginia. Os entrevistados foram recrutados pelas autoridades do 
turismo. A pesquisa abordava a questão da satisfação com a viagem, e discutia se os turistas se consideravam visitantes típicos do referido destino. Os resultados mostraram uma relação positiva entre a auto congruência e satisfação. Assim, os turistas que se consideram visitantes típicos de Nortfolk, reportaram grande satisfação com a viagem.

Com referência às várias fases da auto congruência propostas por Sirgy (1985), Chon (1992) e Litvin e Goh (2003) investigaram se a auto congruência real e a ideal influenciavam na satisfação de turistas queforam para Singapura. Os resultados apontaram que, no nível agregado, alguns visitantes viam Singapura de uma forma mais familiar, ou seja, atrelada à sua forma de ser (auto congruênciareal), e com isso se sentiam mais alinhados com a cidade. No que concerne à forma como eles gostariam de ser vistos (auto congruência ideal), estes também estavam mais satisfeitos em relação ao destino turístico do que aqueles com baixo nível de auto congruência.

Beerli, Menezes e Gil (2007) realizam uma análise distinta da realizada por Livtin e Goh (2003). A partir das análises de regressões, Beerli, Menezes e Gil (2007) evidenciaram que a similaridade entre o auto conceito real e ideal e a imagem do destino possui uma boa tendência para destinos turísticos. Conquanto, este efeito torna-se moderado se o turista já teve uma experiência naquele lugar, reduzindo o efeito da teoria da auto congruência, ou se o indivíduo é altamente identificado com determinado lugar turístico (esse, por sua vez, terá altos índices do efeito da auto congruência).

\section{Métodos}

A partir dos conceitos apresentados na revisão bibliográfica e dos artigos estudados, o tipo de pesquisa mais utilizado para se realizar a mensuração do conceito personalidade do destino é o método de levantamento ou survey. Portanto, seguindo o modelo dos estudos de Usakli e Baloglu (2011), Ekinci e Hosany (2006), Murphy, Moscardo e Benckendorff (2007), o presente trabalhofoi realizado utilizando pesquisa quantitativa, com características descritivas (validação de fatos, estimativas e relacionamentos, representatividade através de amostras e análise estatísticas de dados).

O questionário da presente pesquisa foi aplicado aos alunos em mobilidade estudantil de uma universidade pública do interior do estado de São Paulo. A população de estudantes internacionais nesse campus, no primeiro semestre de 2015, era composta por 151 alunos, sendo 
a faixa etária entre 20 a 54 anos, com $49 \%$ do sexo masculino e $46 \%$ do sexo feminino.Os estudantes eram de diversas nacionalidades, origináriosdoscontinentes Europa, América do Sul, América do Norte, Oriente Médio, África e Ásia.

As questões que os respondentes foram submetidos basearam-se no estudo desenvolvido por Usakli e Baloglu (2011). Esse artigo foi escolhido como pilar para a confecção do questionário, pois abordou os principais pontos propostos nessa investigação científica; personalidade do destino e auto congruência.Na confecção do questionário, foram utilizadas as duas abordagens de auto congruência feitas por Usakli e Baloglu (2011); auto congruência real e ideal. No trabalho de Usakli e Baloglu (2011), na avaliação da auto congruência real, os autores tentaram buscar nas respostas o quanto realmente os entrevistados se achavam parecidos com a personalidade de Las Vegas. Em relação à auto congruência ideal, a intenção foi verificar se os respondentes veem Las Vegas como algo que gostariam de ser comparados, de certa forma, um ideal a ser atingido. A mesma solicitação foi feita nesta pesquisa, porém em relação ao Brasil enquanto destino turístico.

As avaliações por parte dos estudantes estrangeiros sobre a personalidade do Brasil e também sobre a sua auto-congruência com o país foram coletadas por meio da escala Likert de cinco pontos (quanto mais próxima a resposta era de 5, mais os estudantes concordavam com a frase). Há de se ressaltar que, como o questionário não possui zero absoluto na escala, conclusões sobre a magnitude absoluta na medição não poderão ser feitas (MALHOTRA, 1996; MATTAR,1996).

A coleta de dados nesta pesquisa foi realizada por meio de um questionário administrado e respondido via internet, em uma página desenvolvida especificamente para esta função, devido à praticidade do método. As técnicas de análise empregadas foram a análise fatorial exploratória e a comparação de médias.

\section{Resultados e Discussões}

Foram obtidas 95 respostas completas. A média de idade dos respondentes é de 26,08 anos. O respondente mais novo tinha 20 anos e o mais velho tinha 54 anos. A frequência de homens é de $51,6 \%$, enquanto que as mulheres representam $48,4 \%$.

Por meio na análise fatorial exploratória, buscou-se a redução dos dados referentes às questões sobre dimensões da personalidade do Brasil, com o objetivo de posteriormente simplificar nas análises referentes às comparações entre níveis deauto congruência. Foi definido que objetivo da 
realização da análise fatorial neste artigo cientificoera verificar se as variáveis da dimensão da personalidade propostas inicialmente no estudo de Usakli e Baloglu, (2011), seriam representadas neste estudo. Com a proposta da analise fatorial especificada, definiu-se um conjunto de variáveis a ser estudado: as questões referentes às dimensões da personalidade do Brasil.

Com o objetivo de mensurar o ajuste dos dados à análise fatorial, foi aplicado o teste KMO (resultado de 0,792). Segundo Hairet, al (1995), valores de KMO iguais os maiores que 0,80 são considerados excelentes, iguais ou acima de 0,70 são regulares, iguais ou acima de 0,60 são determinados com medíocres, iguais ou acima de 0,50 ruins e abaixo de 0,50 são considerados inaceitáveis.

O método de extração deste estudo consistiu na utilização da análise de componentes principais, uma vez que, nesse ponto o objetivo é reduzir para o número mínimo de fatores, os quais responderão pela máxima variância dos dados do estudo. Com relação ao número de fatores extraídos, foi utilizado o critério deeigenvalues maiores do que 1, na qual apenas os fatores latentes ou eigenvalues maiores do que 1 foram considerados.

Com os critérios escolhidos, foram obtidos 5 fatores com eigenvalues maiores do que 1, que explicam juntos 58,66\% da variância total dos elementos. Os fatores que não correspondem pelo menos 1 de variância, não foram considerados (HAIR et al., 1995). De acordo com Hairet al., (1995), na área de Ciências Sociais, uma solução que represente a partir de 60\% da variância total (em alguns casos, até menos) podem ser considerados como soluções satisfatórias. Para outro autor Boyd, Westfall e Stasch (1989), uma solução que contém a variância total entre $60 \%$ a $70 \%$ é considerada um bom ajustes de dados. Portanto, devido a certa divergência de opiniões, fica a critério do pesquisador decidir quantos fatores serão mantidos nas análises subsequentes. Entretanto, pelo resultado obtido no presente estudo, torna-se plausível assumir como razoável o valor de 58,66\% de variância total explicado, ou seja, mais da metade da variância está sendo explicada pelos fatores observados.

Alinhando com a sugestão de Hairet al., (1995), foi realizada a rotação dos fatores obtidos para as questões que abordavam as dimensões da personalidade do Brasil, usando-se o método Varimax, que é o mais comumente utilizado (MALHOTRA, 1996). Verifica-se na tabela 1 que duas sentenças foram desconsideradas nas análises subsequentes. A primeira é a sentença com o adjetivo "Cheerful", pois a mesma carrega em intensidade semelhante em dois fatores diferentes: $(0,437)$ no fator 2 e $(0,460)$ no fator 1 . Por conseguinte, a sentença com o adjetivo 
"Charming" possui uma fraca relação com os fatores, sendo sua intensidade muito próxima emtrês fatores. No fator 2 sua intensidade é $(0,333)$, fator $4(0,386)$ e por último no fator 5 $(0,444)$. Portanto, esta variável também foi desconsiderada das análises feitas.

O segundo passo na interpretação dos fatores obtidos envolveu avaliar a confiabilidade interna das dimensões, que de acordo Hairet al., (1995), é uma medida da consistência entre múltiplas dimensões de uma variável. O raciocínio subjacente à consistência interna é que os itens individuais de uma escala deveriam medir o mesmo construto, e portanto, ser altamente intercorrelacionados. Nesta pesquisa a consistência interna foi determinada pelo coeficiente alfa de Cronbach, submetendo cada fator encontrado a uma análise de confiabilidade. De acordo com Hairet al.,(1995), o limite inferior para o alfa de Cronbach é de 0,70, no entanto, este pode cair para 0,60 em pesquisas denominadas exploratórias.

O fator 1 é composto por 7 sentenças que caracterizam a personalidade do Brasil por meio dos adjetivos: independent, inteligent, confident, leader, sucessful, uptodate e upper class. Este fator, devido à proximidade com as dimensões identificadas por Usakli e Baloglu, (2011), foi intitulado de a dimensão da "Competência". A variância explicada por esse fator é 30,15\% da variância total dos elementos e possui consistência interna, de acordo com o coeficiente alfa que é igual a 0,864. Dessa forma, determinado fator pode ser considerado confiável, por apresentar um valor de coeficiente alfa superior a 0,70 .

O segundo fator explica 9,712\% da variância total dos dados, e é determinado pelas sentenças relacionadas à personalidade do Brasil que usam os adjetivos:alive, energetic, sexy, vibrant e showy. A consistência interna medida pela alfa de Cronbach é igual a 0,772. A exclusão das sentenças em sua maioria não interfere, ou seja, não melhora o alfa. Entretanto, como no fator anterior há apenas uma sentença em que ocorre uma pequena melhora no alfa, no entanto, a mesma foi mantida, pois a melhora não foi considerada de grande significância para a exclusão de tal sentença. Dessa forma, o fator 2 também pode ser considerado confiável, pois o alfa de Cronbach está acima de 0,70.

O próximo fator, 3 , representa 7,857\% da variância total de todos os elementos analisados, sendo composto 4 sentenças que usam os adjetivos: friendly, imaginative, unique e young. $\mathrm{O}$ valor do alfa de Cronbach neste fator 0,719 , indicando que o fator 3 é confiável. O fator 3 apresenta sentenças que no trabalho de Usakli e Baloglu (2011)integraram a dimensão de “Contemporâneo". Usakli e Baloglu (2011) definem essa dimensão através das seguintes 
sentenças: unique, upto date, imaginative, young e trendy. No presente estudo,upto date foi relacionado à primeira dimensão, que é a "Competência", e trendy pode ser observado em outra dimensão que será evidenciada posteriormente.

O fator 4 explica 5,721\% da variância total dos elementos analisados acerca da personalidade do Brasil, sendo composto por 3 sentenças que usam os adjetivos glamorous, goodlooking e trendy.A consistência interna medida pela alfa de Cronbach é igual a 0,681. Apesar de ser abaixo de 0,70 , o número é bastante próximo ao ideal, sendo assim considerado que a consistência interna do fator 4era suficientemente adequada. $\mathrm{O}$ fator 4 em relação ao trabalho realizado por Usakli e Baloglu (2011), possui um número menor de sentenças e algumas que não foram observadas no presente estudo. No estudo apresentado por Usakli e Baloglu (2011), a dimensão de personalidade intitulada Sofisticação era composta por 5 sentenças que usaram os adjetivos:feminine, charming, upperclass, goodlooking e glamorous. Por sua vez, neste trabalho, o fator 4 apresentou-se com 3 variáveis dispostas na tabela acima. Entretanto, devido à grande pontuação que as sentenças glamorous e goodlooking obtiveram no fator, consequentemente este estudo designou o fator 4 como "Sofisticação".

Tabela 1: Matriz de Rotação das Sentenças da Personalidade do Brasil

\begin{tabular}{l|l|l|l|l|l}
\hline Sentenças & \multicolumn{5}{|c}{ Componentes } \\
\cline { 2 - 6 } & $\mathbf{1}$ & $\mathbf{2}$ & $\mathbf{3}$ & $\mathbf{4}$ & $\mathbf{5}$ \\
\hline Brazil is an Independent Country & 0,738 & 0,237 & & & \\
\hline Brazil is an Intelligent Country & 0,724 & & 0,206 & 0,352 & \\
\hline Brazil is a Confident Country & 0,694 & & & & \\
\hline Brazil is a Leader Country & 0,687 & & & 0,253 & 0,275 \\
\hline Brazil is a Successful Country & 0,683 & 0,216 & 0,230 & 0,310 & \\
\hline Brazil is an Up - to - Date Country & 0,520 & & 0,328 & 0,340 & \\
\hline Brazil is an Upper Class Country & 0,452 & & $-0,268$ & 0,347 & 0,294 \\
\hline Brazil is an Alive Country & 0,202 & 0,718 & & & \\
\hline Brazil is an Energertic Country & 0,203 & 0,689 & & & \\
\hline Brazil is a Sexy Country & & 0,675 & & 0,325 & \\
\hline Brazil is a Vibrant Country & 0,246 & 0,658 & 0,308 & & 0,303 \\
\hline Brazil is a Showy Country & & 0,492 & 0,247 & 0,308 & $-0,448$ \\
\hline Brazil is a Friendly Country & & & 0,727 & & \\
\hline Brazil is an Imaginative Country & 0,227 & & 0,677 & & \\
\hline Brazil is an Unique Country & & & 0,656 & & \\
\hline Brazil is a Young Country & & & 0,637 & & \\
\hline Brazil is a Cheerful Country & & 0,437 & 0,460 & $-0,208$ & \\
\hline Brazil is a Glamorous Country & 0,245 & & & 0,763 & 0,282 \\
\hline Brazil is a Trendy Country & & & 0,295 & 0,674 & \\
\hline Brazil is a Good Looking Country & 0,278 & 0,450 & & 0,509 & \\
\hline Brazil is a Feminine Country & & & & & 0,700 \\
\hline Brazil is a Daring Country & & 0,333 & & & 0,640 \\
\hline Brazil is an Exciting Country & $-0,209$ & 0,430 & 0,392 & & 0,497 \\
\hline Brazil is a Charming Country & & 0,333 & 0,220 & 0,386 & 0,444 \\
\hline
\end{tabular}


O fator 5 representa 5,213\% da variância total dos elementos da personalidade do Brasil, sendo composto por 3 sentenças contendo os adjetivos feminine, exciting e daring. O valor do alfa de Cronbach é 0,555, sendo um pouco abaixo da exigência de confiabilidade de 0,70 do alfa de Cronbach. Segundo De Vellis (1991), em alguns cenários de investigação das ciências sociais, um alfa de Cronbach de 0,60 é considerado aceitável, desde que os resultados obtidos com esse instrumento sejam interpretados com precaução e tenham em conta o contexto de computação do índice em questão. Com isso, considerou-se confiável a consistência interna do fator 5.

O fator 5 deste presente estudo é a principal diferença encontrada em comparação ao estudo de Usakli e Baloglu (2011). Composto das sentenças contendo os adjetivos feminine, exciting e daring, este fator se diferencia do estudo dos autores citados, pois essas sentenças no estudo de Usakli e Baloglu (2011), estão inseridas em diversas outras dimensões. Por conseguinte, analisando a sobreposição das sentenças neste fator, foi concebida a ideia de intitulá-lo como a dimensão "Sensual". A literatura sobre imagem do Brasil enquanto destino turístico indica que o Brasil possui uma conotação sensual e até as vezes sexual frente aos estrangeiros,como evidenciado em Caetano (2004)..Segundo Caetano (2004), nas décadas de 1970-90, a antiga Empresa Brasileira de Turismo investia na imagem do Brasil, associando a mulher brasileira a um atrativo turístico, utilizando seguidamente figuras femininas seminuas, associadas a paisagens naturais (como praias) ou a eventos culturais (Carnaval). Dessa forma, devido à grande importância desta dimensão para este estudo e também pela particularidade do mesmo em relação ao trabalho proposto por Usakli e Baloglu (2011), decidiu-se manter este fator, mesmo com o alfa de Cronbach abaixo do valor ideal.

As cinco dimensões identificadas neste estudo deram origem a cinco variáveis, que foram criadas com base na média das respostas que compõem cada sentença referente àpersonalidade do brasil enquanto destino turístico. A ordenação dessas dimensões pode ser visualizada na Tabela 2.

Tabela 2: Estatísticas Descritivas acerca das Dimensões de Personalidade do Brasil

\begin{tabular}{l|l|l|l|l|l}
\hline Dimensões & $\mathbf{N}$ & Mínimo & Máximo & Média & Desvio \\
\hline Vibração & 95 & 2,20 & 5,00 & 4,0316 & 0,67623 \\
\hline Contemporâneo & 95 & 2,00 & 5,00 & 3,9447 & 0,68434 \\
\hline Sensual & 95 & 1,67 & 5,00 & 3,4702 & 0,75088 \\
\hline Sofisticação & 95 & 1,33 & 5,00 & 3,1439 & 0,79248 \\
\hline Competência & 95 & 1,00 & 4,86 & 2,9188 & 0,68151 \\
\hline N Válido & 95 & & & & \\
\hline
\end{tabular}


A dimensão com a maior pontuação média foi a Vibração. A imagem do Brasil é constantemente associada a denominações que levam a pensar que o paísseja extremamente vibrante. Mariutti e Giraldi (2013) relatam que a imagem do Brasil é relacionada a aspectos positivos, estando ligada ao Carnaval, Futebol, Samba ou pessoas como Pelé, Gisele Bundchen, Ronaldo e Zico. Bignami (2002) divide a imagem do Brasil no exterior em cinco categorias e nelas está incluída a diversidades de atributos e adjetivos que direcionam o país para uma personalidade vibrante. Portanto, a colocação da dimensão "Vibração" em primeiro lugar não se torna surpresa, confirmando uma personalidade da imagem do Brasil bastante difundida entre os estrangeiros.

Logo após a dimensão Vibração, está a dimensão Contemporâneo e, em terceiro lugar, a dimensão Sensual. Tal resultado demonstra a importância desta dimensão, que foi a única com alfa de Cronbach abaixo de 0,6, mas, que, no entanto, está intimamente ligada à avaliação que o estrangeiro faz da personalidade do Brasil. A dimensão Sofisticação está na quarta posição e, em último lugar, a Competência.

Posteriormente, buscou-se verificar como as dimensões aqui identificadas se relacionam com a auto congruência. A mensuração da auto congruência também foi realizada a partir da escala de Likert, em que os entrevistados poderiam atribuir pontuações de 1 "discordo totalmente, até 5 "Concordo Totalmente" com cada sentença. Foram construídas seis sentenças, baseadas no estudo de Usakli e Baloglu (2011), que também abordou a auto congruência separando-as em duas dimensões, auto congruência ideal e auto congruência real.Essas dimensões foram derivadas de estudos prévios conduzidos por Sergy et al. (1997), Sergyand Su (2000) e Helgson e Suphellen (2004).

Dessa forma, três questões foram feitas referentes àauto congruência real e, por conseguinte, três questões foram abordadas em relação à auto congruência ideal. Emrelaçãoà auto congruência real, foram feitas as seguintes questões: "Brazil is consistent with how I see myself", "I am quite similar to personality of Brazil", "The personality of Brasil is congruent with how I see myself". Para a auto congruência ideal, houve as seguintes sentenças: "Brazil is consistent with how I would like to see myself"," I would like to be perceived as similar to the personality of Brazil", "The personality of Brazil is congruent with how I would like to see myself".

Para analisar a confiabilidade das sentenças e consequentemente a convergência das questões referentes àauto congruência, foi utilizado o alfa de Cronbach. Como, segundo Hair et al. 
(1995), um alfa de Cronbach maior do que 0,7 é considerado aceitável, acredita-se que os resultados são confiáveis em nível ótimo, pois o alfa de Cronbach da análise de auto congruência foi de 0,946 .

A auto congruência foi dividida em dois níveis, alta e baixa, com o objetivo de analisar quais são as implicações desses doisníveis da auto congruência nas dimensões referente à personalidade do Brasil aferida pelas respostas dos estudantes estrangeiros. Dessa forma, as frequências que foram encontradas para cada nível dividiram-se da seguinte forma: a baixa auto congruência teve uma frequência de 51 estudantes, representado um percentual 53,7\%. Já a alta auto congruência foi resultado das respostas de 44 alunos intercambistas, com um percentual de 46,3\%. Em suma, há mais estudantes com baixa auto congruência do que o contrário.

Nas tabelas 3 e 4 abaixo se procuraram relacionar as dimensões de personalidade do Brasil com os níveis deauto congruência. Tal fato decorre da perspectiva definida pela teoria da auto congruência, que postula que consumidores tendem a escolher produtos ou marcas que estão mais próximas do seu auto conceito (USAKLI; BALOGU, 2011). Aaker (1995) menciona que a noção básica da teoria da auto congruência é dirigida para consumidores que preferem marcas que sejam congruentes com a sua personalidade.

Tabela 3: Estatísticas descritivas da baixa auto congruência

\begin{tabular}{l|l|l|l|l|l}
\hline Dimensões & N & Mínimo & Máximo & Média & Desvio \\
\hline Vibração & 51 & 2,20 & 5,00 & 3,8275 & 0,69284 \\
\hline Contemporâneo & 51 & 2,50 & 5,00 & 3,6814 & 0,70548 \\
\hline Sensual & 51 & 2,00 & 5,00 & 3,2157 & 0,73582 \\
\hline Sofisticação & 51 & 1,33 & 4,33 & 2,8889 & 0,72316 \\
\hline Competência & 51 & 1,00 & 3,86 & 2,6555 & 0,58287 \\
\hline N Válido & 51 & & & & \\
\hline
\end{tabular}

Tabela 4: Estatísticas descritivas da alta auto congruência

\begin{tabular}{l|l|l|l|l|l}
\hline Dimensões & N & Mínimo & Máximo & Média & Desvio \\
\hline Vibração & 44 & 2,40 & 5,00 & 4,2682 & 0,57860 \\
\hline Contemporâneo & 44 & 2,00 & 5,00 & 4,2500 & 0,51715 \\
\hline Sensual & 44 & 1,67 & 4,67 & 3,7652 & 0,66114 \\
\hline Sofisticação & 44 & 1,67 & 5,00 & 3,4394 & 0,77350 \\
\hline Competência & 44 & 2,14 & 4,86 & 3,2240 & 0,66537 \\
\hline N Válido & 44 & & & & \\
\hline
\end{tabular}

Observando as estatísticas descritivas, há uma atribuição com notas médias maiores em todas as dimensões (Vibração, Contemporâneo, Sensual, Sofisticação e Competência) por parte dos respondentes que foram avaliados como alta auto congruência.Entretanto, é importante 
salientar que mesmo com o aumento das notas em relação às dimensões, não houve mudança nas ordenações das dimensões em relação aos dois grupos de auto congruência, ou seja, a dimensão Vibração continuou em primeiro lugar e a Competência permaneceu em último.

Nessas duas dimensões, há fortes características da personalidade do Brasil. No que se refere à Vibração, podem ser considerados os elementos festas, carnaval, futebol, e praias, que são muito relacionados a essa dimensão. Quanto à dimensão de competência, pode-se citar a corrupção, desorganização e violência, que são fatores que vão contra ao critério de competência.

Diferentemente do estudo de Usakli e Baloglu (2011), procurou-se aqui analisar a auto congruência com relação às dimensões da personalidade que foram assinaladas pelos estudantes estrangeiros, com o objetivo de aferir se a auto congruência afeta na avaliação da personalidade do respondentes sobre o país em questão. Já Usakli e Baloglu (2011) relacionaram a teoria de auto congruência com a intenção de retorno e recomendação do país que o respondente visitou. No próximo tópico serão apresentadas e discutidas as conclusões deste trabalho.

\section{Conclusões}

Esta pesquisa buscou identificar quais são os elementos da personalidade do Brasil mais latentes para um público de estrangeiros, além de verificar qual é a influência da auto congruência na avaliação dos estudantes acerca da personalidade que os mesmos atribuíram ao país. A partir da análise fatorial a respeito das sentenças contendo adjetivos que caracterizam o Brasil enquanto destino turístico, foi possível estabelecer cinco dimensões de personalidade de destino. É importante ressaltar que as dimensões encontradas vieram confirmar as dimensões construídas no estudo de Usakli e Baloglu (2011), o qual este artigo tomou como base para construir o questionário.

As cinco dimensões identificadas com a análise fatorial exploratória deste estudo foram: Vibração, Competência, Contemporâneo, Sofisticação e Sensual. A última dimensão foi a única que se diferenciou em relação ao trabalho original de Usakli e Baloglu (2011).Esta descoberta é a grande contribuição deste estudo. Diferentemente do trabalho realizado por Usakli e Baloglu (2011), houve o surgimento dessa nova dimensão, que é característica da personalidade do Brasil, aparecendo em artigos e levantamentos sobre a imagem do país. Essa dimensão é 
composta por três sentenças relacionadas aos adjetivos feminine, exciting e daring. A forte associação do Brasil com mulheres seminuas nos carnavais, com praias, samba e entre outros são fatores explicadores da constituição dessa dimensão.

Outra importante contribuição desta pesquisa é a confirmação da pouca credibilidade do Brasil no que envolve fatores ligados à competência: essa dimensão apareceu como última na ordenação das associações à personalidade do país. Problemas sociais, como a pobreza, violência, crimes, altos índices de corrupções e falta de infraestruturas em algumas cidades são explicações que condizem com a colocação insatisfatória da referida dimensão. Como a literatura indica que há falta de um conjunto confiável e válido para medição das dimensões da personalidade de país (ROJAS-MÉNDEZ; MURPHY; PAPADOPOULOS, 2013), esta é uma contribuição adicional desta pesquisa.

A partir das pontuações obtidas na análise da auto congruência, os respondentes foram divididos entre aqueles que possuíam baixa auto congruência e alta auto congruência em relação à personalidade do Brasil. Nessa avaliação, constatou-se que os respondentes que apresentavam um nível mais alto de auto congruência atribuíram maiores escores às dimensões propostas do que aqueles que estavam perfilados no grupo de baixa auto congruência.

No entanto, uma ressalva relevante nesta análise se dá pelo fato de que não houve alterações na ordenação das dimensões entre os respondentes de alta e baixa auto congruência, ou seja, mesmo para diferentes níveis de auto congruência ou de identificação com a personalidade do Brasil, as dimensões de personalidade do destino continuam em suas respectivas posições.

Por fim, como implicações práticas desta pesquisa, pode-se sugerir que empresas que possuem ligação com o setor de turismo podem referenciar suas estratégias de comunicação no desenvolvimento de sentimentos positivos em relação ao país (atração, felicidade, interesse, tecnologia, organização, transparência e desenvolvimento), como forma de gerar intenções de visitar o país e desenvolver as dimensões tanto de competências como ratificar as dimensões de vibração. Além disso, agências do governo e profissionais de marketing de diferentes setores industriais podem ajustar seus programas de comunicação, de forma a passar ao exterior uma imagem de Brasil mais competente, que não é somente o país da dimensão sensual e vibrante, mas também um país que possui importantes características que podem contribuir fortemente para o desenvolvimento da imagem de competência frente ao ambiente internacional. 
Em relação às limitações referentes ao método de pesquisa utilizado, duas observações são aplicáveis. A primeira e também a mais significativa limitação é referenteà falta de uma amostragem aleatória. Os respondentes foram escolhidos por critérios de conveniência, ou seja, a amostra não foi do tipo probabilística. Portanto, a amostra escolhida neste estudo não reflete a população de visitantes do Brasil. Uma segunda limitação metodológica advém do método empregado para a coleta de dados (via questionário auto-administrado na forma eletrônica). Este pode ter gerado vieses em relação ao entendimento das questões, mesmo sendo realizados pré-testes para identificar e eliminar possíveis problemas de falta de compreensão.

Quanto às sugestões para estudos futuros, a primeira sugestão é direcionada para um maior desenvolvimento de pesquisas que abordam o conceito de auto congruência relacionada ao setor de turismo. Em segundo lugar, estudos futuros poderiam trabalhar com amostras aleatórias, para os resultados poderem ser generalizados. Finalmente, a dimensão de personalidade do Brasil "Sensual", identificada neste estudo, poderia ser avaliada em outras situações metodológicas (escalas, amostras e análises), com o propósito de verificar se confiabilidade desta dimensão é aceitável e se a mesma se replica em outros públicos de turistas do Brasil.

\section{Referências}

AAKER, J. L. Dimensions of Brand Personality. Journal of Marketing Research, 34:347-56, 1997.

BALOGLU S, MCCLEARLY KW. A model of destination image formation. Annals of Tourism Research,26(4):868-97, 1999.

BEERLI, A., DIAZ, G. and GIL, S. M. Self-Congruity and Destination Choice.Annals of Tourism Research, 34 (3), 571-587, 2007.

BEERLI, A., J. MARTIN Factors Influencing Destination Image. AnnalsofTourismResearch31:657$681,2004$.

BIGNAMI, R.A imagem do Brasil no turismo. 2. ed. São Paulo: Aleph, 2002.

BOYD, H.W.; WESTERFALL, R.; STASCH, S. F. Marketing research: text and cases. 7th ed. Homewood: Irwin, 1989.

CAETANO, R. A publicidade e imagem do produto Brasil e da mulher brasileira como atrativo turístico", in: Anais do $27^{\circ}$ Congresso Brasileiro de Ciências da Comunicação, 27, São Paulo: Intercom, 2004.

CHON, K.-S., (1992).Self-Image/Destination Image Congruity.Annals of Tourism Research, 19 (2), 360-363.

D'ASTOUS, A.; BOUJBEL, L. Positioning countries on personality dimensions: Scale development and implications for country marketing. Journal of Business Research, v.60, n.3, 231-239, 2007.

EKINCI, Y.; HOSANY, S. Destination personality: an application of brand personality to tourism destinations. Journal of Travel Research, v. 45, n. 2, p. 127-139, 2006. 
EPSTEIN, S.The cognitive self, the psychoanalytic self, and the forgotten selves.Psychological Inquiry, v. 3, p. 34-37, 1992.

GARTNER, W. Image Formation Process.Journal of Travel and Tourism Marketing 2:191-215, 1993.

GRAEFF, T. R. Image congruence effects on product evaluations: The role of self-monitoring and public/private consumption. Psychology \& Marketing, v. 13, n. 5, p. 481-499, 1996.

HAIR, J. F.; ANDERSON, R. AND.; TATHAM, R. L.; BLACK, W. C. Multivariate data analysis. Upper Saddle River: Prentice Hall, 1995.

HOSANY, S.; EKINCI, Y.; UYSAL,M. Destination image and destination personality: an application of branding theories to tourism places. Journal of business research. 2006.

JAFFE, E.D.; NEBENZAHL,I.D. National image \& competitive advantage. Copenhagen: Copenhagen Business School Press, 2001.

KIM, S., and X. Y. LEHTO, Projected and Perceived Destination Brand Personalities: The Case of South Korea.Journal of Travel Research, 52:117-30, 2013.

KOTLER, Philip; HAIDER, Donald H.; REIN, Irving.Marketing Places:attracting investment, industry, and tourism to cities, states, and nations, Nova Iorque: Free Press, 1993.

LANDON, E. L. Self-concept, ideal self-concept and consumer purchase intentions. Journal of Consumer Research, v. 1, n. 2, p. 44-51, 1974.

LEE, J., and L. K. XIE.Cognitive Destination Image, Destination Personality and Behavioral Intentions: An Integrated Perspective of Destination Branding. Paper presented at the 16th Graduate Students Research Conference. University of Massachusetts, Amherst, MA., 2011.

LEE, H. J., and Y. G. SUH.Understanding and Measuring City Brand Personality (CPS) in the Context of South Korean Market.International Journal of Tourism Sciences, 11 (3): 1-20,2011.

LITVIN, S. W. and GOH, H. K. Individualism/Collectivism as a Moderating Factor to the Self-Image Congruity Concept. Journal of Vacation Marketing, 10 (1), 23-32, 2003.

MALHOTRA, N. K..Marketing research: an applied orientation. Upper Saddle River: Prentice Hall, 1996.

MALHOTRA, N. K. Self-concept and product choice: an integrated perspective. Journal of Economic Psychology, v. 9, n.1, p. 1-28, 1988.

MARIUTTI, F. G.; GIRALDI, J. M. E.; CRESCITELLI, E. The Image of Brazil as a Tourism Destination: An E xploratory Study of the American Market. International Journal of Business Administration, v. 4, p. 13-22, 2013.

MARIUTTI, F. G.; GIRALDI, J. M. E. Country Brand Identity: An Exploratory Study about the Brazil Brand with American Travel Agencies. Tourism Planning \& Development, v. 11, p. 1-14, 2013.

MATTAR, F.N. Pesquisa de marketing, edição compacta, São Paulo: Atlas 1996

MURPHY, L., BENCKENDORFF, P., MOSCARDO, G. Destination brand personality: visitor perceptions of a regional tourism destination. Tourism Analysis, v. 12, n. 5-6, p. 419-432, 2007.

MURPHY, L; MOSCARDO, G.; BENCKENDORFF, P. Using brand personality to differentiate regional tourism destinations.Journal of Travel Research, vol. 46, no. 5, p. 14.,2007.

NADEAU, J. et al. Destination in a country image context. Annals of Tourism Research, vol. 35, n.1, p.84-106, 2008.

NAGASHIMA, A.A comparison of Japanese and U.S. attitudes towards foreign products.Journal of Marketing, New York, v. 34, p. 68-74, Jan. 1970. 
ONKVISIT, S.; SHAW, J. Self-concept and image congruence: some research and managerial issues. Journal of Consumer Marketing, v. 4, n. 1, p.13-23, 1987.

PAPADIMITRIOU, D.; APOSTOLOPOULOU, A.; KAPLANIDOU, K. Destination personality, affective image, and behavioral intentions in domestic urban tourism.JournalOfTravelResearch, 2013.

PÉREZ-NEBRA, A. R.; ROSA, C. J. As novas estratégias de promoção do Brasil no exterior: estudo de caso. Turismo em Análise, v. 19, n. 3, p. 450-471, 2008.

PRAYAG, G. Exploring the relationship between destination image and brandpersonality of a tourist destination: an application of projective techniques. Journal of Travel and Tourism Research, 111-130, 2007.

REZENDE-PARKER, A. M.; MORRISON, A. M.; ISMAIL, J. Dazed and confused?An exploratory study of the image of Brazil as a travel destination.Journal of Vacation Marketing, v. 9, n.3, p.243-259, 2003.

ROJAS-MÉNDEZ, J.I.; MURPHY, S. A.; PAPADOPOULOS, N. The U.S. brand personality: a sino perspective. Journal of Business Research, v. 66, n. 8, p.1028-1034, 2013.

SAHIN, S., and S. BALOGLU. Brand Personality and Destination Image of Istanbul.Anatolia-An InternationalJournal of Tourism and Hospitality Research, 22:69-88, 2011.

SIRGY, M. J. Self-concept in consumer behavior: a critical review. Journal of Consumer Research, 9, 287-300, 1982.

SIRGY, M. J. Using self-congruity and ideal congruity to predict purchase motivation.Journal of Business Research, 13, 195-206, 1985.

SIRGY, M. J., GREWAL, D., MANGLEBURG, T. F., PARK, J., CHON, K., CLAIBORNE, C. B., JOHAR, J. S., \& BERKMAN, H. Assessing the predictive validity of two methods of measuring selfimage congruence. Journal of the Academy of Marketing Science, 25, 229-241, 1997.

SWANN, W. B., STEIN-SEROUSSI, A. and GIESLER, R. B. Why People Self-Verify. Journal of Personality and Social Psychology, 62 (3), 392-401, 1992.

SIRGY, M. J., JOHAR, J. S., SAMLI, A. C., \& CLAIBORNE, C. B. Self-congruity versus functional congruity: predictors of consumer behavior. Journal of the Academy of Marketing Science, 19(4), 363375, 1991.

TUAN, Y. Images and Mental Maps.Annals of the Association of American Geographers, 65:205-13, 1975.

USAKLI, A., S. BALOGLU.Brand Personality of Tourist Destinations: An Application of SelfCongruity Theory.Tourism Management, 32:114-27, 2011.

VERLEGH, P. W. J.; STEENKAMP, J. B. AND. M.A review and meta-analysis of country of- origin research. Journal of Economic Psychology, Amsterdam, v. 20, Iss.5, p. 521-546, 1999.

\section{Recebido em: 06/11/2015 ( $1^{\text {a }}$ versão) $14 / 01 / 2016$ ( $2^{\mathrm{a}}$ versão) \\ Aprovado em: 12/04/2016}

